\title{
BMJ Open Evaluation of the referral management systems (RMS) used by GP practices in Northumberland: a qualitative study
}

\author{
Rosie Dew, Scott Wilkes
}

To cite: Dew R, Wilkes S. Evaluation of the referral management systems (RMS) used by GP practices in Northumberland: a qualitative study. BMJ Open 2019;9:e028436. doi:10.1136/ bmjopen-2018-028436

- Prepublication history for this paper is available online. To view these files, please visit the journal online (http://dx.doi. org/10.1136/bmjopen-2018028436).

Received 7 December 2018 Revised 6 June 2019 Accepted 10 June 2019
Check for updates

(C) Author(s) (or their employer(s)) 2019. Re-use permitted under CC BY-NC. No commercial re-use. See rights and permissions. Published by BMJ.

School of Medicine, University of Sunderland, Sunderland, UK

Correspondence to

Professor Scott Wilkes;

scott.wilkes@sunderland.ac.uk

\begin{abstract}
Objective Exploring the views of stakeholders to the referral management systems (RMS) used by GP practices in Northumberland, UK to evaluate its perceived effectiveness.
\end{abstract}

Design This was an in-depth qualitative semi-structured interview study.

Participants and setting 32 participants (GPs, hospital consultants, referral support, hospital managers, Clinical Commissioning Group manager) in the North East of England, UK.

Method Interviews using a grounded theory approach and thematic analysis.

Results The main benefit of RMS mentioned by participants was that it allowed for unnecessary referrals to be vetted by consultants, and helps ensure patients are sent to the correct clinic. Generally, the consultants in our study felt that RMS did not significantly help them reject referrals. Some GPs experienced that RMS undermined GP autonomy and did not help when they had exhausted their abilities to manage a patient in primary care, and it was suggested that in some cases RMS may delay rather than prevent a referral. The main perceived disadvantage of RMS was the additional workload for GPs and consultants, and RMS was felt to be a barrier to commutation between GPs and consultants. Frustration with the system design and lack of knowledge of its cost-effectiveness were articulated.

Conclusion Although RMS was reported to reduce some unnecessary referrals, the effect of referral delay and rejection is unknown. Although there were some positive attributes described, RMS was mostly received negatively by the stakeholders.

\section{INTRODUCTION}

Referrals by GPs to another service are the result of around 1 in 20 GP consultations. ${ }^{1}$ In England, nearly 15 million referrals were made from primary care between 2016 and 2017. ${ }^{2}$ Clinical Commissioning Groups (CCGs) are used by the National Health Service (NHS) in England, and hold responsibility for commissioning hospital services and some primary care services. ${ }^{3}$ Overuse of hospital services is contributing significantly to the financial deficit of many CCGs in the UK, with the average avoidable

\section{Strengths and limitations of this study}

There is a lack of knowledge of referral management systems (RMS), and few studies to date have investigated the views of GPS and consultants, and other stakeholders involved in referral management.

- In-depth interviews with the stakeholders involved in RMS provided rich insights into their attitudes to, and experiences of, RMS.

- This was a small study based in the North East of England.

- This study was limited to the views of the stakeholders involved in RMS.

Patient views were not considered in our study.

hospital outpatient appointment costing $£ 145$. ${ }^{4}$ To date, there is no consensus on the definition of an appropriate referral.

There is a wide range of primary care approaches designed to reduce the number of referrals and improve the effectiveness of outpatient services including minor surgery in primary care, direct access by GPs to diagnostic tests, specialist attachment to primary care teams and professional education programmes. ${ }^{5}$ Professional behaviour change approaches including education from specialists and structured referral sheets have been shown to generally reduce GP referrals. ${ }^{6} 7$ The availability of specialist advice by email or phone to primary care practitioners and the use of telemedicine also show potential to reduce referrals. ${ }^{8}$ Moreover, improved quality of referral information, specialist contact prior to referral, electronic referrals and community specialist services have been shown to help address referral demand, ${ }^{9}$ and studies have shown that referral assessment by consultants may increase referral quality and reduce the amount of referrals. ${ }^{10-12}$ However, lack of change on referral rates were seen for strategies using passive distribution of local referral guidelines, ${ }^{79}$ feedback on referral rates and discussion with an independent medical guide. ${ }^{7}$ There is little evidence of 
the benefit of the relocation of specialists to primary care, or joint primary and secondary care management of patients on outpatient referrals rates. ${ }^{8}$ Additionally, financial methods to manage referrals risk inappropriate reductions in referrals and negative impacts on referral quality and patient care. ${ }^{7}$

The electronic referral system, 'Choose and Book', was introduced by the NHS in the UK to allow GPs to book an appointment for a patient in the hospital or clinic of choice, ${ }^{13}$ and is associated with increased efficiency in terms of reduced administrative burden, and non-attendance to outpatient appointments. ${ }^{13} 14$ Electronic referrals have also been found to reduce waiting times to triage. ${ }^{14}$ Moreover, although an increase in referrals was seen, electronic referral management with triage and input from an advanced primary care service lead to reduced waiting times, reduced duplicate referrals and high patient satisfaction. ${ }^{15}$

Referral management systems (RMS) are used to manage referrals from primary care and are a current strategy used by CCGs to reduce the number of referrals and encourage primary care solutions to be found. ${ }^{16}$ CCGs in England spend millions of pounds on RMS each year. ${ }^{17}$ There are a number of RMS including: 1) external appraisal by a referral management centre (RMC), often a private company, 2) local expertise from consultants or GPs with a special interest employed to triage referral letters, 3) specialist advice from consultants to GPs about management of a patient and 4) peer review and reflection where referrals are reviewed by other GPs. ${ }^{16}$

In 2017, 39\% of CCGs in England commissioned an RMS. ${ }^{17}$ There have been few evaluations of RMS and the effects they are having on patients and clinicians are relatively unknown. ${ }^{717}$ A pilot trial of local expertise RMS was found to decrease the number of incomplete referrals, increase adherence to referral guidelines and increase patient satisfaction. ${ }^{18}$ There is evidence to suggest that a referral management and booking service (RMBS) triage process allowed GPs to consider referral guidelines and alternative care options before referring, however, concern was raised regarding technical issues, cost-effectiveness and additional workload. ${ }^{18}$ RMCs may filter out inappropriate referrals, direct referrals to a more appropriate setting or accelerate the diagnostic pathway. However, RMCs may also cause an increase in the overall cost, ${ }^{19}{ }^{20}$ cause misdirected referrals due to lack of full clinical information, reduce close working between GPs and consultants, demotivate local GPs and lead to delayed or lost referrals. ${ }^{19}$ A retrospective time-series analysis of outpatient attendances in one primary care trust in England found that the use of an RMC did not reduce the hospital outpatient attendance rate or attendance rate ratio, and in some situations increase both. ${ }^{21}$ Moreover, the British Medical Association (BMA) raised concern that RMS are a short-term approach to healthcare, and suggested that there is need for more evaluation. ${ }^{22}$

There is little and variable evidence supporting referral schemes reducing referrals ${ }^{5}$ and/or costs. ${ }^{5}{ }^{23}$ When considering the cost-effectiveness of referrals, a whole systems approach has rarely been studied. We do not understand how professionals navigate the barriers and enablers of RMS, and the attitudes and perceptions of GPs, patients, specialists and commissioners have been relatively unexplored. The NHS faces a potential funding gap of £14-20 billion, and taking into account that GPs take the lead for commissioning services, ${ }^{19}$ examining whether RMS can help GP commissioners reduce costs and improve care by monitoring the impact of RMS is needed.

'Consultant First' (CF) is the electronic RMS used by Northumberland CCG that replaced 'Choose and Book'. Referrals are triaged by local consultants, and if further investigations, alternative dispositions or clinical management advice are considered appropriate, then the referral will be returned to the referring GP with management advice from the consultant for discussion with the patient, otherwise the patient will be allocated an outpatient appointment. CF was agreed with the main provider, Northumbria Healthcare NHS Foundation Trust (NHCFT), as an alternative to an RMS implemented by many other CCGs. CF covers 14 specialties all of which, apart from dermatology (which is provided by Newcastle Hospitals NHS Foundation Trust), is provided by NHCFT. All 41 GP practices in Northumberland use CF. This study sought to understand the attitudes and experiences of professionals involved in the RMS in Northumberland, to investigate the advantages and disadvantages of CF and to identify any unintended consequences that are associated with its use.

\section{METHODS}

\section{Study design}

This qualitative study ran from August 2017 to March 2018. In-depth interviews were used to explore the experiences and attitudes of GPs, consultants, commissioners, hospital managers and referral support staff towards the RMS used by GP practices in Northumberland, UK.

\section{Participants and sampling}

Using purposive sampling, ${ }^{24}$ suitable participants with GP, consultant or commissioning roles involved in RMS were identified and recruited via email through Northumberland CCG. Thereafter, theoretical sampling, ${ }^{25}$ recruiting key participants, was used until themes from the interview data were saturated.

Thirty-two participants took part in a one-off interview, of which 15 were GPs, 10 were hospital consultants, 3 had referral support roles, 2 were hospital managers, 1 was a CCG manager and 1 was a part-time GP with a CCG role (table 1). Patients were not recruited to our study since we sought to investigate the benefits and challenges perceived by those using RMS.

Interviews were conducted by the female research associate (RD) at the participant's place of work, although one GP was interviewed at their home, and three GPs and 
Table 1 Participant characteristics

\begin{tabular}{|c|c|c|c|c|c|c|}
\hline ID & Job role & Age range (years) & Sex & $\begin{array}{l}\text { Years in } \\
\text { current role }\end{array}$ & $\begin{array}{l}\text { GP practice } \\
\text { list size }\end{array}$ & $\begin{array}{l}\text { GP practice } \\
\text { referral rates }\end{array}$ \\
\hline GP1 & GP & $30-35$ & M & 3 & Medium & Low \\
\hline GP2 & GP & $46-50$ & M & 21 & Small & Low \\
\hline GP3 & GP & $41-45$ & M & 15 & Large & Low \\
\hline GP4 & GP & $46-50$ & M & 7 & Medium & High \\
\hline GP5 & GP with CCG role & $41-45$ & M & 14 & Large & Low \\
\hline GP6 & GP & $46-50$ & M & 20 & Large & Low \\
\hline GP7 & GP & $41-45$ & M & 15 & Medium & Low \\
\hline GP8 & GP & $41-45$ & M & 16 & Medium & Low \\
\hline GP9 & GP & $46-50$ & $\mathrm{~F}$ & 23 & Large & Low \\
\hline GP10 & GP & $46-50$ & M & 18 & Small & Low \\
\hline GP11 & GP & $46-50$ & M & 21 & Medium & High \\
\hline GP12 & GP & $36-40$ & $\mathrm{~F}$ & 17 & Large & Low \\
\hline GP13 & GP & $36-40$ & $\mathrm{~F}$ & 12 & Medium & High \\
\hline GP14 & GP & $56-60$ & $\mathrm{~F}$ & 33 & Large & Low \\
\hline GP15 & GP & $41-45$ & $\mathrm{~F}$ & 15 & Medium & High \\
\hline GP16 & GP & $51-55$ & $\mathrm{~F}$ & 24 & Small & High \\
\hline C1 & Orthopaedic surgeon & $41-45$ & M & 8 & $\mathrm{n} / \mathrm{a}$ & $\mathrm{n} / \mathrm{a}$ \\
\hline $\mathrm{C} 2$ & Rheumatologist & $41-45$ & M & 5 & $\mathrm{n} / \mathrm{a}$ & $\mathrm{n} / \mathrm{a}$ \\
\hline C3 & Consultant gastroenterologist & $51-55$ & M & 22 & $\mathrm{n} / \mathrm{a}$ & $\mathrm{n} / \mathrm{a}$ \\
\hline $\mathrm{C} 4$ & Cardiologist & $56-60$ & M & 32 & $\mathrm{n} / \mathrm{a}$ & $\mathrm{n} / \mathrm{a}$ \\
\hline C5 & Colorectal surgeon & $56-60$ & M & 33 & $\mathrm{n} / \mathrm{a}$ & $\mathrm{n} / \mathrm{a}$ \\
\hline C6 & Respiratory physician & $41-45$ & M & 21 & $\mathrm{n} / \mathrm{a}$ & $\mathrm{n} / \mathrm{a}$ \\
\hline $\mathrm{C7}$ & Colorectal surgeon & $51-55$ & $\mathrm{~F}$ & 31 & $\mathrm{n} / \mathrm{a}$ & $\mathrm{n} / \mathrm{a}$ \\
\hline $\mathrm{C} 8$ & Rheumatologist & $36-40$ & $\mathrm{~F}$ & 3 & $\mathrm{n} / \mathrm{a}$ & $\mathrm{n} / \mathrm{a}$ \\
\hline C9 & Gastroenterologist & $46-50$ & M & 14 & $\mathrm{n} / \mathrm{a}$ & $\mathrm{n} / \mathrm{a}$ \\
\hline $\mathrm{C} 10$ & Respiratory physician & $41-45$ & M & 19 & $\mathrm{n} / \mathrm{a}$ & $\mathrm{n} / \mathrm{a}$ \\
\hline M1 & CCG manager & $51-55$ & M & 4 & $\mathrm{n} / \mathrm{a}$ & $\mathrm{n} / \mathrm{a}$ \\
\hline M2 & Contact centre lead & $56-60$ & $\mathrm{~F}$ & 1 & $\mathrm{n} / \mathrm{a}$ & $\mathrm{n} / \mathrm{a}$ \\
\hline M3 & Contact centre manager & $56-60$ & $\mathrm{~F}$ & 12 & $\mathrm{n} / \mathrm{a}$ & $\mathrm{n} / \mathrm{a}$ \\
\hline M4 & Appointments coordinator & $56-60$ & $\mathrm{~F}$ & 7 & $\mathrm{n} / \mathrm{a}$ & $\mathrm{n} / \mathrm{a}$ \\
\hline M5 & Hospital assistant manager & $41-45$ & $\mathrm{~F}$ & 8 & $\mathrm{n} / \mathrm{a}$ & $\mathrm{n} / \mathrm{a}$ \\
\hline M6 & Hospital manager & $51-55$ & $\mathrm{~F}$ & 11 & $\mathrm{n} / \mathrm{a}$ & $\mathrm{n} / \mathrm{a}$ \\
\hline
\end{tabular}

Practice list size: small 0-4999; medium 5000-9999; large 10 000+. $\mathrm{n} / \mathrm{a}$, not available.

one consultant had telephone interviews. Due to participant time availability, one focus group was held with two hospital managers and an appointments office coordinator, while the rest of the participants were interviewed alone. Six GPs, two consultants, two directorate managers and three participants with referral support roles were female, while the remaining participants were male. Participants' age ranged from 30 to 59 years, and the number of years participants had been working in their current role ranged from 1 to 33 years (table 1). Participants did not have an established relationship with RD; however, two participants had taken part in a previous study with the researcher.

Five GP interviewees were from practices that had referral rates that were higher than the Northumberland CCG average for 2017-2018, and the remainder were from practices with referral rates lower than the CCG average. The practice list size ranged from 2051 to 23328 patients. In 2018, the mean size of practices in Northumberland CCG was 7742 and practice sizes ranged from 1595 to 23328 . Five GPs reported that they worked parttime in general practice, three GPs described they were 
Box 1 Initial topic guide used to explore the attitudes and experiences of stakeholders regarding consultant first

\section{Initial topic guide for interviews}

How would you describe your experience with referrals?

Are you aware of any system to help manage referrals?

What do you think about Consultant First?

Would you make any changes to Consultant First? What changes?

What do you think are the positive aspects of Consultant First?

What do you think are the negative aspects of Consultant First?

Do you receive/provide follow-up information if a referral has been ac-

cepted or had an alternative disposition? If so, what information?

Have you ever experienced a situation where it was felt that the patient

did not need to be seen? If so why?

Do you know how many referrals are alternative dispositions?

Have you experienced delays in referrals as a consequence of Consultant First/RMS?

What do you think about the use of triage in referrals by consultants in the specialty you are referring to?

What do you think about the use of triage in referrals by GPs?

Have referral management systems caused any problems for you, the specialist or the patient? If so, what problems?

Does Consultant First/RMS provide useful educational feedback to the referrer? If so, how?

Is Consultant First an appropriate tool to manage resource in a cash-limited NHS? Why?

Is there anything else you would like to discuss?

NHS, National Health Service; RMS, referral management systems.

part-time GPs but worked full-time hours, two consultants worked part-time, while all the remaining participants worked full-time in their roles.

\section{Analysis}

A grounded theory approach ${ }^{24}$ was used and a semi-structured topic guide was employed to explore the study's aims. The initial topic guide was informed by issues raised in the literature, and gaps of knowledge regarding CF, and is shown in box 1 . The questions asked were adjusted appropriately according to the participant (GP, consultant or other). Interviews were audio-recorded and transcribed verbatim, except for one GP interview where written notes were taken and no audio was recorded at the request of the participant. Interviews lasted $42 \mathrm{~min}$ on average and participants were not paid for their time and transcripts were not returned to participants. RD did not have medical training or experience using CF, and a neutral approach to analysis was conducted to encourage confirmability of the data analysis and establish trustworthiness. Data analysis and theme negotiation was conducted by RD and SW.

Using an inductive thematic analysis, ${ }^{26}$ open coding of interview data was initially performed using Microsoft Word, and axial coding followed grouping codes into categories and finally into overarching themes using iterative constant comparison in Microsoft Excel. ${ }^{25}$ Interview themes were developed from the data analysis and were used in subsequent interviews to guide questioning and the topic guide was developed as the interviews proceeded ${ }^{27}$ until no new themes emerged and data were saturated. ${ }^{25}$

\section{Patient and public involvement}

Patients or public were not involved in this study.

\section{RESULTS}

Four themes emerged from the interviews: GP professional control and responsibility, consultant risk aversion and accountability, the burden of RMS and the perceived benefit of RMS (figure 1).

\section{GP professional control and responsibility \\ GP autonomy}

In this study, most GPs were from GP practices that took part in an internal peer-review process of all potential referrals. It was generally considered that CF did not significantly reduce referrals further. GPs described managing patients within primary care to avoid unnecessary referrals through $\mathrm{CF}$, and GPs with special interests or knowledge in a particular condition/s helped improved management of patients within primary care.

I think if you took away referral management... Internal referral management, the number of consultations that Consultant First has to triage would rocket. (GP3)

We have lots of education based on people's special interests as well. ...You know, to try and avoid referrals, if we can. (GP12)

Alternative routes that GPs can use to avoid CF to ensure a referral is accepted were mentioned, including other referral pathways and trauma clinic services:

Newcastle do MSK (musculoskeletal), and Northumbria do MSK. There's nothing stopping a GP referring direct to Newcastle and avoiding Consultant First. (M1)

Some GPs felt that CF undermines GP autonomy and referral rejection through $\mathrm{CF}$ could have negative effects on GPs including preventing GPs from referring similar appropriate cases in the future, and affecting relationships with patients following shared decision making:

And, actually, it may put them off making similar referrals in the future, which may be appropriate. (GP7)

You go through something with a patient and you take your time doing it. And you get to the management at that point, and then you sort of do a shared decision about where to go next. And then it almost feels like... We've just been undermined. (GP11)

It was suggested that $\mathrm{CF}$ may delay rather than prevent referrals in cases when GPs have followed advice provided by consultants through $\mathrm{CF}$ and then re-referred. Moreover, some GPs felt that if a necessary referral had been 


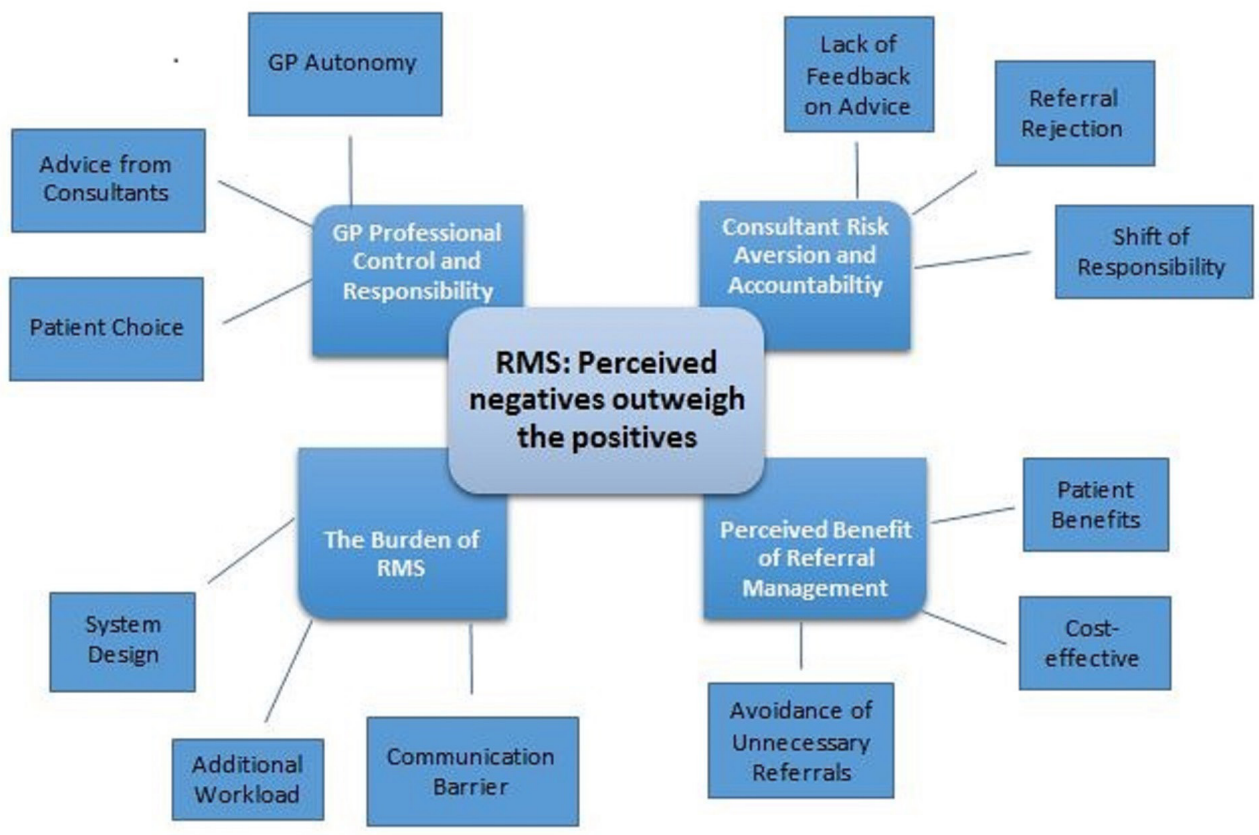

Figure 1 Concept diagram of the interview themes. RMS, referral management systems.

rejected, CF did not prevent referrals being rewritten and resubmitted:

I don't think it's always stopped me from referring, because I had patients where I've done those things. They're still abnormal and I've referred them back. So, I don't think it's particularly stopped a referral. So, it hasn't done the purpose it's there for. (GP13)

The easiest thing to do is just to re-write that letter and use different trigger words. (GP2)

CF did not help some GPs manage patients in primary care who request a second opinion from a consultant, or situations where the GP had requested a referral as they felt that they had exhausted their abilities to manage a patient in primary care. Additionally, two consultants suggested that rejecting a referral that was requested to meet patient demand may actually be less cost-effective than accepting the referral request:

And there's a risk with this system that they can write back and say, 'Well, do this, this and this'. And you think, that's all very well, but it actually doesn't help me. I still feel out of my depth and need your help and assistance to manage this patient. (GP7)

And although the GPs have had to pay us $£ 150$ to... To carry that out, the patient is happy. And maybe we knock ten GP appointments out of the next 12 months. (C9)

Advice from consultants provided through RMS

GPs mentioned that, although the response was often returned quickly, the advice provided through CF by consultants was mostly appropriate, but was often limited, and it was felt by some GPs that the advice quality was variable depending on the replying consultant. The majority of GPs felt that the advice through CF generally did not contribute directly to improving GP knowledge or understanding:

So, sometimes quite useful. Sometimes really not helpful at all. (GP16)

And there definitely are, from different consultants, different sort of standards of advice. (GP9)

So, it's not educational. It's more just matter of fact. (GP13)

GPs recounted that they were unsure who receives their referral requests through $\mathrm{CF}$ and felt that since the advice provided through CF was anonymous, they did not have a reference point within secondary care. GPs also recounted that, on the contrary to the 'Choose and Book' system used previously, they are not provided feedback on whether a patient's referral is accepted:

I'm not sure whether then that letter will go to that consultant, or will just go through a triaging consultant... (GP3)

No, we don't hear anything. Whereas, when we doing referrals through Choose and Book, at least we would know when they would be seen. (GP3)

\section{Patient choice}

Some GPs felt that the lack of choice for patient appointments was an issue with CF compared with 'Choose and Book'. It was also mentioned that CF referral system had caused confusion for some patients that were expecting an outpatient appointment, but instead advice only was provided to the GP: 
It takes away choice-which patients have been used to with the Choose and Book system. (GP15)

But I then say, well, you might just get an appointment. And that leads to some confusion and definitely some patients not turning up, I suspect. (GP12)

\section{Consultant risk aversion and accountability}

Referral rejection

CF was reported to reject around 3\%-10\% of referrals. However, even with CF in place, the majority of consultants felt that they could not reject many referrals, due to non-specific symptoms or the nature of the specialty:

I think, really, from our point of view, the main negatives are that we don't feel we've been able to bounce anything like the number that I think the CCG were hoping for. (C8)

And there's an awful lot of undifferentiated symptoms, as well, that get referred in. Like breathlessness and coughing. (C10)

\section{Lack of feedback on advice provided}

In general, consultants felt that they did not know whether the advice they provide through CF was helpful to GPs as they had not been provided with any feedback within the system. Moreover, feedback on whether they are using the system correctly and the cost-effectiveness of CF was also suggested by GP and consultants:

To be able to get an idea about, you know, if we have given advice, was the advice useful? (C4)

I would be interested to see how much money it saved. And how many referrals it saved. (C10)

\section{Shift of responsibility}

The shift of responsibility of referral management through CF from primary to secondary care was mentioned by some participants. Consultants felt that $\mathrm{CF}$ allowed them to make the decision about which patients they see in their clinics, and on the contrary to GP perceptions, gave them more control compared with the "Choose and Book' system previously used:

At least with this system, the consultant shares some of the responsibility by looking at the letter and deciding how quickly they need to be seen, and actually if their appointment slot isn't appropriate, prioritising them. (GP7)

However, some consultants felt that since the appointment times were managed externally, $\mathrm{CF}$ had resulted in them having less control over patient appointment slots, in some cases causing delays for urgent appointments:

If there's a routine slot that's earlier than that, they won't allocate the person to the routine slot. ...So, they will just stick with it's triaged as urgent, we'll go for the next urgent slot. ...So, you can get delays. (C2)

\section{The perceived benefits of RMS}

Avoidance of unnecessary referrals

One main advantage of CF mentioned by participants was that it allowed for unnecessary referrals to be vetted by consultants, and helps ensure patients are sent to the correct clinic the first time:

Well, I think the positive aspects are that for $6 \%$ to $10 \%$ we're managing to avoid a referral. (C3)

There's always that, sort of, triaging these things, so that they go to the right place first. (GP11)

\section{Perceived patient benefits}

The patient benefits of CF were also mentioned by participants, including the potential to save a number of appointments for patients and reducing waiting times:

Less appointments, less cost, less hassle for the patients. (GP10)

I think particularly for, sort of, patient safety, it offers the opportunity to look at a letter and go, oh, hang on-we should probably see that sooner... (C1)

\section{Cost-effectiveness}

Some participants perceived that CF was a cost-effective method of managing referrals, including potentially avoiding unnecessary referrals, and only paying for an administration fee compared with other RMS used in the NHS:

I guess there's perceived advantages for cost saving in that if you look at a patient's letter and go, oh, I don't see why there's any point in seeing me, then they get diverted to a less costly service. (C1)

Whereas this has just got an admin fee, as you mentioned. And apart from that, it's free. (GP5)

However, some GPs and commissioners felt there is a financial incentive for consultants to accept referrals through CF and therefore the hospital to charge the CCG for an outpatient appointment. On the contrary, consultants and hospital managers remarked that instead the focus was on managing their workload:

$£ 110, £ 120, £ 130 \ldots £ 200$-odd. For some outpatient appointments. So why would they say, 'I don't need to see you'. (M1)

And I can honestly say that our clinics are so busy, and our waiting lists are so long, that we would rather turn work away. (C9)

\section{The burden of RMS}

\section{Additional workload}

The additional workload as a result of CF was a problem raised by both GPs and consultants, including increased time spent on triage in secondary care, patient management in primary care following referral rejection and administrative work for both: 
I've got to find some time to ring the patient. I've got to do some more administration, do another referral letter. (GP6)

We don't have a great deal of time, and it's extra work. (C10)

Since the majority of consultants worked in a number of hospitals, the electronic nature of CF meant consultants could access the system in different hospitals. However, consultants perceived the advice they provided through $\mathrm{CF}$ was basic due to time constraints. Most consultants felt that the advice requests through $\mathrm{CF}$ were increasing and further funding to support consultant time and depth of advice in the future may be required:

So, it's quicker. I can access this from any computer in... In the Trust. (C2)

Really low level (advice). Really, kind of, as a consequence of how much time we've got to offer. (C9)

It's become an advisory service, not just a referral management service, and that will... We will need to change what we do if that grows more and more, because it will mean more consultant time, and actually... it needs properly funding. (C4)

\section{Communication barrier between GPs and consultants}

Due to the one-way electronic nature of the system, CF was felt by some to be a barrier to communication between primary and secondary care, and did not encourage relationship development between GPs and consultants as no back and forth conversation could be achieved through the system:

But there's no way of getting in touch with a GP if they send a referral in and you think, oh my God, they need to do something about this now....You end up spending half an hour on the phone, trying to get past the administrators. (C9)

\section{System design}

Frustration with CF software was felt by most participants, including slow processing, issues trying to open and link attachments and lack of integration with other systems used. A few consultants raised concern that when using $\mathrm{CF}$ there is no option to go back to a referral to check or change it after an action has been made:

...It can't come through into System One, which is the system that we use. (GP15)

And they (contact centre) would move people, on the patient's request, but then that wouldn't feed back into our system, because the IT wasn't integrated. (C5)

I can't bring that referral back, you know... So, I'm sure I've accepted things purely by mistake. (C4)

Some GPs and commissioners recounted an issue with the 'dummy slot number' used in the system that changes from when the referral is first requested in primary care to when an appointment has been made by the contact centre. Problems such as the GP practice not knowing the new number and confusion for patients were mentioned:

So, if the GP books this dummy slot... Which is always between midnight and two o'clock in the morningif they print that out and give it to the patient, it's got this address on. It might say, 'Your appointment is 1 am'. The patient thinks, well, that's just a mistake. And they turn up here at one o'clock in the afternoon. (M3)

Improvements to CF suggested by participants included integrated test requests for consultants to select, digital dictation for advice provided by consultants, in-house control of appointment slots by consultants and notification of a patient's appointment provided to the GP:

If we took back, within house, our admin team-allocated appointments. (C2)

It would just be a bit useful to have some notification of when the patient is going to be seen. (GP3)

\section{DISCUSSION \\ Principal findings}

It was generally considered that CF did not significantly reduce the number of referrals further than internal peer review within GP practices. Some GPs felt that CF undermined GP autonomy and did not help when they had exhausted their abilities to manage a patient in primary care. Variable advice provided through CF from consultants, little knowledge of the triage process and lack of control over patient appointment slots was also mentioned by GPs. It was suggested that CF may lead to additional work for GPs and delays in referrals, including re-referral after first following management advice from consultants, or referrals being rewritten and resubmitted to ensure acceptance. Moreover, consultants mentioned the lack of control of urgent appointment slots in secondary care can cause referral delays.

Generally, the consultants in our study felt that CF did not significantly help them reject referrals, and that there was a lack of feedback on whether they are using the system correctly or its cost-effectiveness. However, it was felt that they had more control over appointment slots than previously.

The main benefit of CF mentioned by participants was that it allowed for unnecessary referrals to be vetted by consultants, and helps ensure patients are sent to the correct clinic. The patient benefits perceived by our participants included reduced appointments and waiting times, while the foremost disadvantage of $\mathrm{CF}$ was the additional workload. CF was also felt to be a barrier to communication between GPs and consultants, and frustration with the system design was expressed. Although the benefits of CF perceived by our participants were mentioned, the perceived disadvantages outweighed the 
advantages, and for the most part stakeholders were sceptical and dissatisfied with CF.

\section{Comparison with the literature}

Similar to CF, the removal of the gatekeeper role for referrals from primary care has been seen in RMCs and has been suggested to have a negative impact on GP autonomy. ${ }^{519}$ However, studies have also shown GPs may choose to defer to the experience of a specialist. ${ }^{19}$ Undermining the doctor-patient relationship is an issue in RMS when shared decisions making between GP and patient is overridden by secondary care decision. ${ }^{28}{ }^{29}$ Moreover, the workload associated with triaging referrals in $\mathrm{CF}$ was an issue present in a Norwegian qualitative study investigating the referral process, ${ }^{30}$ and a British study exploring RMBS. ${ }^{18}$ There is need for the evaluation of the cost-effectiveness of the increased workload in primary care and the community in a resource-limited NHS in the long term. ${ }^{18}$

Poor communication between primary and secondary care has been reported for inflammatory bowel disease, ${ }^{31}$ cancer, ${ }^{32}$ dyspepsia, colonic malignancy, chest pain and chronic obstructive pulmonary disease, ${ }^{33}$ and CF was not perceived to increase communication. However, quick electronic advice was associated with $\mathrm{CF}$ and is highly valued by GPs, ${ }^{29}$ and email communication between primary and secondary care was found to provide quick answers for advice or reassurance and enhance patient benefits such as unnecessary referrals. ${ }^{5-8}{ }^{34}$ However, our GPs did not describe the advice through CF as helpful, and this was similar to the GP perceptions of RMBS. ${ }^{18}$

Problems with the system design of CF were stated by our participants, and technical issues including the functionality and usability of RMBS has been previously cited ${ }^{18}$ as has system design and compatibility issues of an RMC. ${ }^{5}$ It was suggested that CF causes delays, and referral centres have also been proposed to be a possible cause for referral delays ${ }^{19}$; furthermore, the BMA have described RMS to have the potential to harm patients by delaying their management. ${ }^{28}$ Additionally, RMCs have been perceived to focus on cost saving rather than patient care, and calls to have them abolished have been implemented. ${ }^{1628}$ However, CF was perceived to have patient benefits including reducing the number of appointments and waiting times, which has also been identified as a benefit of RMS. ${ }^{1415} 19$

CF allows for specialist advice from local consultants, and follows internal peer review in some GP practices, which are recommendations for referral support schemes stated by the Royal College of General Practitioners, ${ }^{1116}$ and 'in-house' second opinion/peer review before referral has the potential to reduce referrals. ${ }^{679}$

Lack of feedback on whether the system is being used correctly, whether the advice provided by consultants is helpful and little knowledge of the cost-effectiveness of CF was explained by our participants, and was also described by GP participants regarding RMBS previously. ${ }^{18}$ Finally, there is a general lack of understanding of RMS since they are diverse in nature and only a small proportion of RMS have been or are being evaluated. ${ }^{17}$

\section{Strengths and limitations}

Since this study involved a sample of 32 participants from the North East of England, and reports only their experiences and opinions of the benefits and issues with RMS, these data may not be generalisable outside of the study sample. However, this study gathered multiple perspectives and the data captured is rich and reached saturation within the participant sample.

It was a challenge to recruit participants and most participants were male, thus interviews with more female GPs and consultants may have provided a different perspective on RMS. Although all GP surgeries that use CF, and a large proportion of consultants who are involved in the triaging process were invited to participate, those that choose to take part may have had different views from those who were not recruited. Additionally, patient views were not explored in this study. Comparing the perspectives of stakeholders in different specialities, and exploring patient views, who often drive the referral process, would provide further insights.

This study may also have been subject to interviewer bias, however the interviewer did not have medical training or experience of using RMS, and two researchers were involved in the data analysis. Notwithstanding the above limitations, this study is one of a few to evaluate the use of RMS in the UK and provides novel insights into the perceptions of its stakeholders.

\section{Implications for future research or practice}

A successful RMS requires feedback to consultants and GPs, evidence of patient safety and quality management as well as system cost-effectiveness.

Since consultants reported that they only had the capacity to provide basic advice through $\mathrm{CF}$, funding to support more in-depth or increasing advice requests may be required in the future. Our participants made several suggestions to improve RMS including the ability to recall a referral after an action has been taken, in-house control of appointment slots by consultants, notification of a patient's appointment provided to the GP, integrated test requests for consultants to select, digital dictation and the use of a unique reference number.

Evaluating the patient experiences of RMS would provide additional insights, and further evaluation of, and comparison with the RMS used by different CCGs in the UK and internationally would improve the overall understanding of RMS.

Acknowledgements The authors would like to thank all participants who that took part in this study, and those from Northumberland CCG who helped with recruitment.

Contributors SW and RD designed the study. RD gained research skills through attendance at a qualitative interview training course at Newcastle University, and from working on previous qualitative research projects. RD was responsible for the recruitment and interviewing of participants, and wrote the first draft of this paper. $\mathrm{RD}$ and SW were involved in the in-depth data analysis and development of themes 
that emerged from the data, the overall explanation of the data and revision of the final manuscript.

Funding The study was funded by Northumberland Clinical Commissioning Group (CCG).

Competing interests None declared.

Patient consent for publication Not required.

Ethics approval Ethical approval was obtained from University of Sunderland Ethics Research Group. Governance approval was obtained from the Health Research Authority.

Provenance and peer review Not commissioned; externally peer reviewed. Data sharing statement No data are available.

Open access This is an open access article distributed in accordance with the Creative Commons Attribution Non Commercial (CC BY-NC 4.0) license, which permits others to distribute, remix, adapt, build upon this work non-commercially, and license their derivative works on different terms, provided the original work is properly cited, appropriate credit is given, any changes made indicated, and the use is non-commercial. See: http://creativecommons.org/licenses/by-nc/4.0/.

\section{REFERENCES}

1. Foot C, Naylor C, Imison C. The quality of GP diagnosis and referral: The King's Fund. 2010. https://www.kingsfund.org.uk/sites/files/kf/ Diagnosis\%20and\%20referral.pdf

2. NHS Digital. Hospital Outpatient Activity, 2016-17: First attendances 2017. https://digital.nhs.uk/data-and-information/publications/ statistical/hospital-outpatient-activity/hospital-outpatient-activity2016-17

3. NHS England. Clinical Commissioning Groups (CCGs). https://www. england.nhs.uk/ccgs/

4. NHS NECS. Referral Management System (RMS). 2015. http:// monkseatonmedical.nhs.uk/referral-management-system-rms/

5. Winpenny W, Miani C, Pitchforth E, et al. Outpatient services and primary care: scoping review, substudies and international comparisons. HS\&DR 2016;4.

6. Roland M, McDonald R, Sibbald B. Outpatient services and Primary Care: A Scoping Review of Research Into Strategies For Improving Outpatient Effectiveness and Efficiency. National Institute for Health Research Trials and Studies Coordinating Centre: Southampton, 2006.

7. Akbari A, Mayhew A, Al-Alawi MA, et al. Interventions to improve outpatient referrals from primary care to secondary care. Cochrane Database Syst Rev 2008;4:CD005471.

8. Winpenny EM, Miani C, Pitchforth E, et al. Improving the effectiveness and efficiency of outpatient services: a scoping review of interventions at the primary-secondary care interface. J Health Serv Res Policy 2017;22:53-64.

9. Blank L, Baxter S, Woods HB, et al. What is the evidence on interventions to manage referral from primary to specialist nonemergency care? A systematic review and logic model synthesis. HS\&DR 2015;3.

10. Evans E. The Torfaen referral evaluation project. Qual Prim Care 2009;17:423-9.

11. Evans $\mathrm{E}$, Aiking $\mathrm{H}$, Edwards $\mathrm{A}$. Reducing variation in general practitioner referral rates through clinical engagement and peer review of referrals: a service improvement project. Qual Prim Care 2011;19:263-72.
12. Patterson V, Humphreys J, Chua R. Email triage of new neurological outpatient referrals from general practice. J Neurol Neurosurg Psychiatry 2004;75:617-20.

13. Walford S. Choose and Book. Clinical Medicine 2006;6:473-6.

14. Dennison J, Eisen S, Towers M, et al. An effective electronic surgical referral system. Ann R Coll Surg Engl 2006;88:554-6.

15. Maddison P, Jones J, Breslin A, et al. Improved access and targeting of musculoskeletal services in northwest Wales: targeted early access to musculoskeletal services (TEAMS) programme. BMJ 2004;329:1325-7.

16. Royal Collage of General Practioners. Quality patient referrals. Right service, right time. 2018. http://www.rcgp.org.uk/policy/rcgp-policyareas/referral-management.aspx

17. lacobucci G. Referral management schemes: good for whom? BMJ 2017;356:i6856.

18. Wright E, Hagmayer Y, Grayson I. An evidence-based referral management system: insights from a pilot study. Prim Health Care Res Dev 2015;16:407-14.

19. Imison C, Naylor C. Referral management.Lessons for success: The King's Fund. 2010. https://www.kingsfund.org.uk/sites/files/kf/ Referral-management-lessons-for-success-Candace-Imison-ChrisNaylor-Kings-Fund-August2010.pdf

20. Mashta O. Costly referral management centres fail to deliver savings BMJ 2010;341:c4351.

21. Cox JM, Steel N, Clark AB, et al. Do referral-management schemes reduce hospital outpatient attendances? Time-series evaluation of primary care referral management. Br J Gen Pract 2013;63:e386-e392.

22. lacobucci G. Private firms run a third of CCGs' schemes to screen GP referrals. BMJ 2017;356:i6855.

23. Pretty I, Tickle M, Goldthorpe J, et al. An evaluation of a Referral Management \& Triage System for Oral Surgery referrals from primary care dentists: A Mixed Methods study. HS\&DR 2018;6.

24. Strauss J, Corbin A. Basics of qualitative research. Grounded theory proceedures and techniques. Newbury Park: Sage Publications, 1990.

25. Glaser B, Strauss A. The Discovery of Grounded Theory. Chicago: Aldine, 1967.

26. Braun V, Clarke V. Using thematic analysis in psychology. Qualitative Research in Psychology 2006;3:77-101.

27. Denzin NK, Lincoln YS. Handbook of Qualitative Research. London: Sage Publications, 1994.

28. Rimmer A. BMA annual meeting: The BMA will lobby for the abolition of referral management systems. BMJ 2017;357:j3090.

29. Thorsen O, Hartveit M, Baerheim A. General practitioners' reflections on referring: an asymmetric or non-dialogical process? Scand J Prim Health Care 2012;30:241-6.

30. Thorsen O, Hartveit M, Baerheim A. The consultants' role in the referring process with general practitioners: partners or adjudicators? a qualitative study. BMC Fam Pract 2013;14:153.

31. Torjesen I. Care of IBD patients compromised by poor communication between primary and secondary care. BMJ 2012;344:e2675.

32. Bethell GS, Leftwick P. Views of general practitioners and head and neck surgeons on the referral system for suspected cancer: a survey. $J$ Laryngol Otol 2015;129:893-7.

33. Wåhlberg H, Valle PC, Malm S, et al. Practical health co-operation - the impact of a referral template on quality of care and health care co-operation: study protocol for a cluster randomized controlled trial. Trials 2013;14:7.

34. Sampson R, Barbour R, Wilson P. Email communication at the medical primary-secondary care interface: a qualitative exploration. Br J Gen Pract 2016;66:e467-e473. 